\title{
An examination of the research priorities for a hospice service in New Zealand: A Delphi study
}

\author{
KAY DE VRIES, PH.D., M.SC., R.N., ${ }^{1}$ JO WALTON, PH.D., M.A., R.N., ${ }^{2}$ \\ KATHERINE NELSON, PH.D., M.A., R.N., ${ }^{3}$ AND RHONDDA KNOX, M.A., R.N. ${ }^{4}$ \\ ${ }^{1}$ The University of Brighton, Westlain House, Brighton, United Kingdom \\ ${ }^{2}$ Victoria University of Wellington, Wellington, New Zealand \\ ${ }^{3}$ Graduate School of Nursing Midwifery \& Health, Victoria University of Wellington, Wellington, New Zealand \\ ${ }^{4}$ Independent Management Consultant-Health and Public Sector, Wellington, New Zealand
}

(ReCeived May 6, 2015; AccePted May 18, 2015)

\begin{abstract}
Objectives: Palliative care research is relatively diverse and prioritizing research in this field is dependent on multiple factors such as complex ethical decisions in designing and conducting the research; access to participants who may be deemed "vulnerable" and an increasingly medically focused approach to care. The aim of this study was to inform organizational decision-making and policy development regarding future research priorities for a hospice service in New Zealand.

Methods: A modified three-round Delphi technique was employed. Participants were drawn from one dedicated specialist palliative care service that delivers care in the community, daycare, hospice inpatient, aged residential care, and acute hospital palliative care service. A purposive sample included palliative care staff ( $n=10,18,9$, for rounds $1-3$, respectively) volunteers $(n=10,12,11)$; and patients and family carers $(n=6,8$, for rounds 1 and 2$)$. Patients and family carers were not involved in the third round.

Results: At final ranking of six research themes encompassing 23 research topics were identified by staff and volunteers. These were: symptom management; aged care; education; community; patient and family; and bereavement support and young people. Patients and family carers agreed on four themes, made up of 10 research topics. These were: decision-making, bereavement and loss, symptom management; and recognition of need and response of service.

Significance of results : The study generated a rich set of research themes and specific research topics. The perspectives of staff and volunteers are significantly different from those of patients and family members, in spite of the recognition by all concerned that palliative care services work within a philosophy of patient-centered care. Open discussion of ideas has the potential to engage both staff and patients and carers in quality improvement initiatives, and to reinforce the value of research for patient care.
\end{abstract}

KEYWORDS: Research priorities, Palliative care, Hospice staff and volunteers, Family members, Delphi technique

\section{INTRODUCTION AND BACKGROUND}

In general, the goal in health care is to recommend treatments based upon research (i.e., the care should be evidence-based). The same applies for palliative care practice and medicine. Historically, the accumulation of evidence for palliative care has been largely

Address correspondence and reprint requests to: Kay De Vries, University of Brighton, School of Health Sciences, Westlain House, Falmer, Brighton, East Sussex, United Kingdom, BN1 9P. E-mail: 1.k.devries@brighton.ac.uk based on clinical practice and experience of physicians/medical staff (Kaasa \& De Conno, 2001; Kaasa et al., 2006). Most studies conducted in palliative care have been small and descriptive, often without the necessary quality to inform evidence based medicine (Kaasa \& De Conno, 2001; Kaasa et al., 2006; Kaasa \& Radbruch, 2008; Bennett et al., 2010). It has also been argued that palliative care research is not very well developed and that there is no consensus on how to measure the most important outcomes 
within palliative care study populations (Kaasa et al., 2006; Bennett, et al., 2010).

Randomized trials are the gold standard method for evaluating treatments and services in health care, however, they are often difficult to complete in palliative care, and encounter poor recruitment (Higginson \& Booth, 2011) due to perceived vulnerability, moral appropriateness, consent, gate-keeping and inclusion, and the research culture (Duke \& Bennett, 2010). The quality of research in this field is dependent on complex ethical decisions in designing, conducting and reporting this research while addressing concerns for the protection of the safety and rights of people facing life-threatening illness (Duke \& Bennett, 2010; Whiting \& Vickers, 2010).

It is also claimed that the palliative patient population is too ill and too vulnerable to allow meaningful scientific research and that the population is too heterogeneous for clinical research on a group level (Kaasa \& De Conno, 2001). In the case of a dying person, who may not be alive at the end of the research, the judgments made by the researcher may be unchallenged and patients may be unaware of the extent to which the material they have consented to will be used (de Raeve, 1994). Furthermore, there have been reports of adverse effects from what was considered a "low impact" survey of people with life-limiting illness where the content caused significant distress and anxiety among participants (Braithwaite et al., 2009).

Despite the concerns and arguments discussed above, surveys of people with a life-limiting illness who have been asked about participation in clinical trials have demonstrated that they are as likely or more likely than the general patient population to want to be involved in clinical trials (White et al., 2008; White \& Hardy, 2010; Currow et al., 2011). Palliative patients, even those very near death, have been found to be keen to take part in research, both qualitative and quantitative (Ross \& Cornbleet, 2003; Henderson et al., 2005; Pautex et al., 2005; Terry et al., 2006). Terry et al. (2006) found the shared reasons patients wanted to participate in research were: altruism; enhancement of a sense of personal value; the assertion of persisting autonomy; and the value they placed on a commitment by clinicians to optimizing care through research. These reasons are consistent with the views of Berry (2004) when discussing guidelines for ethical research and vulnerable patients. Berry argues that to label palliative care patients as a "vulnerable" population is paternalistic, discriminatory, unjust, and a potential barrier to carrying out important research that can improve the quality of care for dying patients and their families.

\section{PRIORITISING RESEARCH IN PALLIATIVE CARE}

Attempts to prioritize palliative care research are not new. Bennett et al. (2010) reviewed challenges in the delivery of research in end-of-life care in the United Kingdom (UK) and internationally. The review drew on relevant policy and research examples from countries similar to the UK to define some of the issues. They concluded that there were problems of terminology in the field of palliative care and that there was a lack of emphasis on clinical studies relating to the medical aspects of palliative care. Future priorities identified by the authors included the need to build research capacity, make better use of existing research study outcomes, and develop sustained programs of research. They recommended that future research needs to focus on services and public health issues such as understanding care models, evaluating services, surveying social attitudes, and changing cultures (Bennett et al., 2010). However, Bennett et al. (2010) point out that this is problematic in that such research is often conducted after policy initiatives have been put in place, making it difficult to understand the effectiveness and cost effectiveness of new models of care, or for innovation to lead to sustained changes in practice.

In a discussion paper on palliative care research, priorities and the way forward, Kaasa and Radbruch (2008) point out that the study population are described and defined differently across studies and there is no consensus on how to measure the most important outcomes, such as symptoms such as pain, fatigue and depression in palliative care research. These authors proposed that palliative care research should be conducted through the creation of permanent research posts and that these need to have sufficient and sustained funding and be collaborative with other centers of research. They also recommend that as the majority of palliative care patients come from oncology, it is important to establish a close collaboration between these two disciplines.

In summary, there has been considerable clinical research undertaken to improve care for palliative patients and bibliographic databases hold sizeable repositories of palliative care research that pose challenges for professionals in managing the complex evidence-base for this diverse field (Tieman et al., 2009). The aim of this Delphi study was to: inform organizational decision-making and policy development regarding future research priorities; and inform the wider community of the research intentions for a hospice in New Zealand.

\section{STUDY DESIGN}

A modified three-round Delphi technique was used following the methodology described by Malcolm 
et al. (2009). The Delphi technique has been used to identify research priorities in a number of fields, such as the care of infants, children and adolescents (Rudolph et al., 2009; Wilson et al., 2010), midwifery (Fenwick et al. 2006), and palliative care (Malcolm et al., 2008; 2009; Steele et al., 2008; Sigurdardottir et al., 2010). Delphi has also been used in a variety of areas, including government, medical, environmental and social studies, as well as business and industrial research.

The Delphi technique is a structured group communication method that uses a series of questionnaires or "rounds" to gather information or expert opinion from stakeholders about complex problems or novel ideas (Boulkedid et al., 2011). Rounds are held until group consensus is reached. Using this technique allows a large number of individuals across diverse locations and areas of expertise to be consulted simultaneously and anonymously, avoiding domination of the consensus process by one or a few experts (Boulkedid et al., 2011). In this research a modified Delphi procedure, with focus group interviews and meetings and questionnaire Delphi rounds using a nominal group process, was used (Boulkedid et al., 2011). Analysis was integrative to the data collection process. Ethical approval to conduct the study was gained from the Central Regional Ethics Committee.

\section{SAMPLE}

A purposive sample of staff $(n=10)$, volunteers $(n=10)$, patients and family members $(n=6)$ were invited from across the hospice and participated in Round 1 of the study. Recruitment of patients and family members was problematic due to the advanced disease of potential recruits and sensitivity to bereavement issues of family members. Consequently, the number of patient and family member participants was smaller than anticipated. This type of difficulty in recruitment has been demonstrated in other studies involving palliative patients and family members (e.g., Higginson \& Booth, 2011). Community professionals were invited to the focus group interviews but none were able to attend. However, community linked professionals $(n=3)$ provided written lists of areas which they considered a priority for palliative care research.

\section{DATA COLLECTION AND ANALYSIS PROCESSES}

\section{Focus Groups: Round 1}

Round 1 of the Delphi consisted of focus group interviews. These were conducted in three separate groups: hospice staff (doctors, nurses, therapists, social workers); volunteers; and patients and family members together. Participants were provided with an information sheet detailing the study, and written informed consent was obtained from all participants. Questions were developed from the literature and used as an interview guide. In addition, free discussion was encouraged within the groups. These discussions and participant interactions were captured using audio-recording equipment, which were transcribed by a professional transcriber.

A process of repeated reading of the transcripts was carried out by three of the researchers. Themes identifying areas of research interest were classified under a coding system (Table 1).

\section{Focus Groups: Round 2}

A large number (128 items) of research interests were identified and included in the Round 2 questionnaire. All respondents were asked to rate the relevance of each topic on a five-point Likert-type scale, with " 1 " indicating least important and " 5 " indicating most important. Round 2 included some individuals who were not in the Round 1 focus groups. Respondents were also invited to provide further comments on research topics. Each group (staff, volunteers, and patients and family members) received the same

Table 1. Areas of research interest

- Bereavement issues

- Children and young person services

- Therapies and therapists

- Community and primary care

- Cultural considerations

- Ethics and decision-making

- Symptom management

- Education
- Service collaboration/integration

- Continuity of care

- Financing and fund raising

- Patient and family expectations

- Hospice environment

- Spiritual care

- The "good death' concept"
- Increased complexity of patients

- Long-term/chronic conditions

- Medicalisation of hospice

- Confidential conversations

- Sensuality/sexuality issues

- Service capacity

- Aged care 
questionnaire. A unique identifier was used to establish responses by group as literature has clearly demonstrated that health related priorities are very different for professionals than for patients and families. We were uncertain as to whether there would be differences between professionals and volunteers, and sought to explore this in our analysis. Thirty questionnaires were given out to each group $(n=90)$ questionnaires in all. The response rate was: staff $(n=18$, response rate $60 \%)$, volunteers $(n=12$, response rate $40 \%)$, and patients and family members $(n=8$, response rate $27 \%)$, with a total response rate of $42 \%$.

All 38 Delphi responses could be used; however some respondents missed one or more questions. Responses for each question were entered into the Statistical Package of Social Sciences Version 19 and analyzed using descriptive statistics. For each question, the proportion of scores of four or more were calculated, following which all questions were ranked to identify the 48 most preferred topics. The ranking demonstrated that staff and volunteers prioritized similar topics. Consequently, ranking of topics by these two groups were collated for the Round 3 questionnaire.

\section{Focus Groups: Round 3}

The questionnaire developed for Round 3 was administered to staff and volunteers only. Patients and family members were not included in this round due to the limited response from Round 2 and to difficulty in accessing potential participants. Questionnaires ( $n=30)$ were sent out. These were again separated and identifiers used for each of the groups, staff
( $n=15)$ and volunteers $(n=15)$. The questionnaire contained the same topics for both groups but this time a seven-point Likert-type scale was used, with " 1 " indicating least important and " 7 " indicating most important. This broader scale was used to minimize the known propensity of Likert scale responses to center around the midpoint.

The response rate was: staff $(n=9$, response rate $30 \%$ ), and volunteers ( $n=11$, response rate $37 \%$ ), overall response rate of $33 \%$. The analysis followed a similar process to that in Round 2, with the exception that the focus was on scores of six or more. The final priority list was amalgamated for staff and volunteers.

\section{FINDINGS}

\section{Research Priorities for Patients and Family Members}

Priorities for patients and family members were markedly different to those of staff and volunteers. These were categorized into four topic areas: decision-making; bereavement and loss; symptom management; and recognition of need and response of service (Table 2). Within the decision-making category the priorities identified was to examine informed consent and decision-making processes in relation to care practices and to explore how patients were supported by the team in terms of personal preferences and planning for the future. Priorities for bereavement and loss focused on how patients experienced the impact of not being able to participate in activities and hobbies they had previously enjoyed. Research priorities in regard to symptom

Table 2. Topics identified by patients and family member/caregivers

\section{Decision-making}

\section{Bereavement and loss}

Symptom management

Recognition of need and response of service
Examine informed consent and decision-making processes prior to carrying out care in the Hospice (e.g., pre-catheterization; terminal sedation).

Examine how patients are supported to make decisions about how they want to be cared for and planning for the future.

Find out about the sense of loss for patients in not being able to participate in activities and hobbies they have previously enjoyed, e.g., tramping; dancing.

Explore how family member/caregivers manage side effects of medication when caring for palliative patients at home.

Identify how responsive the service is to patients and family member/caregivers.

Look at what "dying well" means from the family member/caregivers and professional perspective.

Explore ways to increase the level of "continuity in the care" of patients and decrease the number of contacts with multiple staff.

Examine how well family member/caregivers are recognized and supported in their care for patients.

Explore the importance of maintaining independence and self-management from a patient's view.

Identify things that patients look forward to, and the goals or things they want to achieve. 
management were on examining side effects of medication when caring for dying patients at home.

For patients and family members, the most significant list of priorities was related to recognition of need and response of services. The list included: identify how responsive the service is to patients and family members/caregivers; examine what "dying well" means from the family and professional perspective; explore ways to increase the level of "continuity in the care" of patients and decrease the number of contacts with multiple staff; examine how well family members/caregivers are recognized and supported in their care for patients; explore the importance of maintaining independence and self-management from a patient's view; and identify things that patients look forward to and the goals or things they want to achieve. This list speaks to the emphasis that the family member/caregivers gave to maintaining their autonomy throughout the terminal illness trajectory.

\section{Research Priorities for Staff and Volunteers}

Research priorities for staff and volunteers were very similar and were considered together. These were categorized into six topic areas: symptom management; aged care; education; community; patient and family; and bereavement support and young people (Table 3). Symptom management was highly prioritized and focused on: development of assessment tools for anxiety associated with breathlessness and sleeplessness (as a symptom cluster); conducting systematic reviews on interventions, care and best practice for each of the main symptom issues in palliative care; find out from patients what level of information they receive about symptoms and ways to manage them, indication of priority to be responsive to family member/caregiver needs; and examine medicalization/pharmacology versus therapeutic interventions in management of symptoms in hospice practice.

The topic of aged care focused on evaluation of the role of the hospice in providing education and clinical support to Aged Care facilities and exploring the preparedness of such facilities for the increase in palliative patients due to an ageing population, particularly in relation to people with dementia. This education theme continued with staff and volunteers prioritizing research into the experiences of staff providing family member/caregiver counselling and examining the education needs of those staff members, and to explore factors for retaining and building the volunteer workforce for the hospice.

Community topics were firmly focused on general practice activities. Priorities were to investigate what general practices and primary health nurses expect from a specialist palliative care program and what the support and education needs of these professionals are. Also included in the priorities of staff and volunteers was the examination of effective partnering with other providers and specialists in the care of palliative patients. Staff and volunteers did include the need to prioritize research on support needs of patient and family however their focus was towards how family members/caregivers manage side effects of medication when caring for palliative patients at home; finding out what it is like for family members/caregivers to have responsibility for monitoring patient changes and adjusting medications in the home; and exploring aspects of trust with health professionals and the hospice when there has been a misdiagnosis. The topic of bereavement was directed at evaluating services and supports for adolescents with palliative care needs.

\section{DISCUSSION}

Of interest, and consistent with the literature, this study has demonstrated differences between research priorities of professionals and volunteers and patients and their families. However, to some extend the broad context of these topics represented the "how" to achieve the "what" outlined in the patient family member/caregivers priorities above so were not completely unrelated but rather were overlapping. The study shows that professional research priorities are primarily focused on evaluation of clinical outcomes and education, upheld in research priority studies of professionals, particularly medical professionals (Sigurdardottir et al., 2010; Daveson et al., 2011). For patients and family members, the research needs focused on research into supportive needs of this group and this is upheld by others (Hudson et al., 2011; Hudson \& Payne, 2011). The small extant literature on research using Delphi or similar methods to investigate research priorities in palliative care, have generally separated the groups. However, a critical examination of this literature has consistently highlighted significant gaps with regard to palliative care research associated with family caregivers (Hudson et al., 2010, 2011). Hudson et al. (2011) set out to determine research priorities in a web-based survey of 55 family caregivers from 12 countries (response rate, $70 \%$ ) who had previously expressed interest in, or were members of The International Palliative Care Family Carer Research Collaboration. Similar to the topics identified in our Delphi study the key areas identified by the caregivers as warranting research attention were: under researched family member/caregiver subpopulations (e.g., young caregivers, ethnic minorities); bereavement experience and support, assessing unmet needs; and development of methods (e.g., assessment 
Table 3. Topics identified by staff and volunteers

\section{Symptom management}

Aged care

Education

Community

Patient/family

\section{Bereavement and support for young people}

Develop a tool that measures anxiety associated with breathlessness including all causes of breathlessness in advanced illness.

Conduct systematic reviews on interventions, care and best practice for each of the main symptom issues in palliative care and update the Hospice palliative care guidelines.

Examine anxiety, breathlessness and sleeplessness (as a symptom cluster) and their impact on patients and family member/caregivers during palliative care and bereavement.

Find out from patients what level of information they receive about symptoms and ways to manage them.

Examine medicalization/pharmacology versus therapeutic interventions in management of symptoms in hospice practice

Evaluate the Specialist Palliative Care Nurse Specialist role in providing education and clinical support to Aged Care facilities.

Examine practice changes in Aged Care Facilities following education of staff on how to use "as required" medications at end-of-life.

Explore the preparedness of Aged Care facilities for the potential increase in palliative care patients due to an ageing population.

Explore hospice practices in caring for people with dementia at the end of life and identify education and training needs of staff.

Investigate the experiences of staff providing family member/caregiver counselling who are not formally trained as Counsellors and examine what the education needs of these staff members are.

Explore factors for retaining and building the volunteer workforce for the future in hospice.

Find out what general practices and primary health nurses expect from a specialist palliative care program.

Investigate the support and education needs of general practices for provision of palliative care in primary health.

Examine what makes effective partnering with other providers and specialists in the care of palliative patients.

Develop a public campaign with volunteers and staff that reflects an image of what hospice actually is, the patient groups cared for (e.g., not just cancer), and the focus of the Hospice work within the community.

Explore ways to increase the level of "continuity in the care" of patients and decrease the number of contacts with multiple staff.

Explore the support needs of family member/caregivers of people who are dying.

Examine the balance between when nursing visits are needed by patients and when patients would like to be independent, with support when they want it.

Explore the aspect of trust with health professionals and the hospice when there has been a misdiagnosis.

Find out what it is like for family member/caregivers to have responsibility for monitoring patient changes and adjusting medications in the home.

Explore how family member/caregivers manage side effects of medication when caring for palliative patients at home

Find out what services and supports are available for adolescents with palliative care needs and what, if any, gaps exist.

Explore what bereavement is for a young person, what coping strategies they use and where they find support. tools) to identify family caregivers at risk of poor psychosocial well-being (Hudson et al., 2011).

Grande et al. (2009) drew on a comprehensive review of the caregiver literature and a consensus meeting by experts in the field to conduct an overview of research on palliative caregivers needs. They considered evidence on: adverse effects of caregiving and interventions to address these; the gaps and weaknesses in the evidence; and how to improve the evidence base and interventions in the future. The authors concluded that there was a lack of longitudinal studies on psychological and bereavement issues and challenges that could have uncovered changes in needs over time, the precursors of increased need, appropriate timing of interventions and the consequences of a failure to meet needs.

In a two phase study, Perkins et al. (2007; 2008) set out to identify research priorities of palliative care patients through focus group interviews and a survey with day therapy and hospice in-patients with cancer 
whose estimated prognosis was six months or less. Of note, and supported by the findings of this study, particularly during the focus group interviews, the researchers found that patients spent most of the time in the groups talking about their own illnesses and how they had affected them and their families. Some participants were able to discuss prioritizing, however most wished to talk more about their own experiences and on what would have improved their own disease journey. They found that symptoms were mentioned infrequently and discussions were more on communication about symptoms, doctors listening more to patients, and patients finding the right form of words to express how they are feeling. These were also emphasized by the patients and family members who participated in this study, with a particular emphasis on wanting to remain in their own homes until death and have autonomy in relation to making decisions and choices about care.

Other groups of professionals and clinicians involved in palliative care practice have set out to determine research priorities. These have included palliative care social workers (Kramer et al., 2005), and providers of palliative care for children (Malcolm et al., 2008; 2009; Steele et al., 2008). The priorities identified by these groups have generally taken a patient and caregiver focus. Social workers identified that there was a need to address mental health needs, grief, bereavement and psychosocial aspects of well-being, cultural issues and humane care, particularly for the most vulnerable and oppressed members of society (Kramer et al., 2005). Research priorities for children were heavily focused on increasing awareness of and improving access to children's hospice care, hospice and respite care needs of young people, and community/home care and issues related to supporting the wider family (Malcolm et al., 2008; 2009; Steele et al., 2008). Research priorities identified by hospice organizations (Tolley \& Payne, 2008) have been more mixed. Tolley and Payne (2008) found that some of the lack of hospice organization participation in research could be attributed to philosophical differences between hospice care and mainstream medicine where the hospice (as a movement) promotes compassionate care for the dying while participation in research may be thought to impinge on this care.

In an attempt to address collaboration, Hagen et al. (2006) conducted a symposium with administrators from the United Kingdom National Cancer Research Institute, the Institute of Cancer Research of the Canadian Institutes of Health Research, and the USA National Cancer Institute. They discussed the feasibility of supporting multinational efforts to strengthen research activities in supportive, palliative, and end-of-life care. Their objective was to cata- lyze high-quality research that would improve this component of care for patients with cancer or other chronic illnesses. The symposium concluded that there was urgent need for research in: measuring symptoms and symptom clusters; study of the biologic bases of complex symptom clusters; enhancing research in symptom interventions; family and informal care-giving; the impact of poverty, societal beliefs and approaches to death and end-of-life care; measuring quality of life within different cultural milieus; performance of the health care system; understanding and responding to transitions in care objectives; and the use of technology to enhance care, and knowledge transfer (Hagen et al., 2006). This comprehensive proposal reflects the priorities of both groups of participants in our study.

\section{STUDY LIMITATIONS}

This study is limited in two aspects. First, it was conducted at only one hospice site however 30 staff and volunteers participated in the study which is an acceptable number for a Delphi study. The second limitation is that patient and family member/caregiver recruitment was low with only eight participants. The reasons for this are consistent with the literature where patients and family member/caregivers declined to participate for reasons such as: fatigue; family member gate-keeping, for example in some cases patients were willing to participate but were prevented by family members.

\section{IMPLICATIONS FOR PRACTICE AND FUTURE RESEARCH}

There are a number of implications for practice and future research that emerge from this study, despite the limitations. Primarily, as palliative care services are steeped in an ethos of patient-centered care it is unsurprising to find that there is some overlap between the views of patients and family members and those of staff and volunteers. Nevertheless, this study demonstrates that important insights into opportunities for quality improvement can be gained through talking with members of the wider health care team (including patients and family members) and making sure that the voices and needs of patients and families are heard.

We were to some degree surprised that volunteers were more aligned to the priorities of professionals than of patients. However, there are multiple factors that may have influenced volunteers having similar research views as professional staff, notwithstanding the complex mix of people who volunteer in hospice and this is an area that requires further examination. 
The small number of patient and family member recruits also meant that we could not separate the two into analyzable groupings. We suggest this as a fruitful area for future research.

\section{CONCLUSION}

The study demonstrated clearly that research priorities identified by staff and volunteers were similar in focus, while those of patients and carers are targeted toward different aspects of palliative and hospice care. Because of the differences in perspective we have kept the two lists of research priorities separate. However, while the two lists are not entirely separate, staff and volunteers focus on education and service provision and symptom management, while patients and family members were predominantly concerned with support with a strong emphasis on maintaining autonomy at all levels of engagement with the palliative care services.

It is entirely consistent with the literature that professional and volunteer workers have a different viewpoint from those for whom they care. It was not surprising that each group focuses on their own area of concern, and indeed we suggest that they may not each know what the gaps in knowledge of the other group are. Patients, for example, will not know what professionals do not know about symptom management, while those who work in the service cannot know exactly what concerns family and caregivers may have over and above those disclosed during care episodes consequentially both sets of priorities are important. A challenge for research funding bodies and palliative care services is to ensure that research that patients and caregivers deem important is also conducted.

\section{ACKNOWLEDGEMENT}

The authors would like to acknowledge Te Omanga Hospice, Upper Hutt, New Zealand for funding and supporting this project

\section{REFERENCES}

Bennett, M.I., Davies, E.A. \& Higginson, I.J. (2010). Delivering research in end-of-life care: Problems, pitfalls and future priorities. Palliative Medicine, 24, 456-461.

Berry, S.R. (2004). For purposes of research, palliative care patients should not be considered a vulnerable population. Clinical Oncology, 16, 223-224.

Boulkedid, R., Abdoul, H., Loustau, M., Sibony, O. \& Alberti, C. (2011). Using and reporting the Delphi Method for selecting healthcare quality indicators: A systematic review. PLoS ONE, 6, e20476.

Braithwaite, M., Philip, J., Finlayson, F., Tranberg, H., Gold, M., Kotsimbos, T. \& Wilson, J. (2009) Adverse events arising from a palliative care survey. Palliative Medicine, 23, 665-669.

Currow, D.C., Agar, M.R. \& Abernethy, A.P. (2011). Tackling the challenges of clinical trials in palliative care. Pharmaceutical Medicine, 25, 7-15.

Daveson, B.A., Harding, R., Vanden Berghe, P., Edwards, S. \& Higginson, IJ. on behalf of PRISMA. (2011). The PRISMA Symposium 4: How should Europe progress end-of-life and palliative clinical care research? Recommendations from the proceedings. Journal of Pain and Symptom Management, 42, 511-516.

de Raeve, L. (1994) Ethical issues in palliative care research. Palliative Medicine, 8, 298-305

Duke, S. \& Bennett, H. (2010). A narrative review of the published ethical debates in palliative care research and an assessment of their adequacy to inform research governance. Palliative Medicine, 24, 111-126.

Fenwick, J., Butt, J., Downie, J., Monterosso, L. \& Wood, J. (2006). Priorities for midwifery research in Perth, Western Australia: A Delphi study. International Journal of Nursing Practice, 12, 78-93.

Grande, G., Stajduhar, K., Aoun, S., Toye, C., Funk, L., Addington-Hall, J., Payne, S. \& Todd, C. (2009). Supporting lay carers in end of life care: current gaps and future priorities. Palliative Medicine, 23, 339-344.

Hagen, N.A., Addington-Hall, J., Sharpe, M., Richardson, A. \& Cleeland, C.S. (2006). The Birmingham International Workshop on supportive, palliative and end-oflife care research. Cancer, 107, 874-881.

Henderson, M., Addington-Hall, J.M. \& Hotopf, M. (2005). The willingness of palliative care patients to participate in research. Journal of Pain \& Symptom Management, $29,116-118$.

Higginson, I.J. \& Booth, S. (2011). The randomized fasttrack trial in palliative care: Role, utility and ethics in the evaluation of interventions in palliative care? Palliative Medicine, 25, 741-747.

Hudson, P. \& Payne, S. (2011). Family caregivers and palliative care: Current status and agenda for the future. Journal of Palliative Medicine, 14, 864-869.

Hudson, P., Zordan, R. \& Trauer, T. (2011). Research priorities associated with family caregivers in palliative care: International perspectives. Journal of Palliative Medicine, 14, 1-5.

Hudson, P.L., Remedios, C. \& Thomas, K. (2010). A systematic review of psychosocial interventions for family carers of palliative care patients. BMC Palliative Care, $9,17$.

Kaasa, S. \& De Conno, F. (2001). Palliative care research. European Journal of Cancer, 37, 153-159.

Kaasa, S., Hjermstad, M.J. \& Loge, J.H. (2006). Methodological and structural challenges in palliative care research: How have we fared in the last decades? Palliative Medicine, 20, 727-734.

Kaasa, S. \& Radbruch, L. (2008). Palliative care research Priorities and the way forward. European Journal of Cancer, 44, 1175-1179.

Kramer, B.J., Bern- Klug, M. \& Francoeur, R.B. (2005). A national agenda for social work research in palliative and end-of-life care. Journal of Palliative Medicine, 8 , $418-431$.

Malcolm, C., Forbat, L., Knighting, K. \& Kearney, N. (2008). Exploring the experiences and perspectives of families using a children's hospice and professionals providing hospice care to identify future research priorities for children's hospice care. Palliative Medicine, 22, 921-928. 
Malcolm, C., Forbat, L., Knighting, K. \& Kearney, N. (2009). Prioritisation of future research topics for children's hospice care by its key stakeholders: A Delphi study. Palliative Medicine, 23, 398-405.

Pautex, S., Herrmann, F.R. \& Zulian, G.B. (2005). Is research really problematic in palliative care? A pilot study. Journal of Pain and Symptom Management, 30, 109-111.

Perkins, P., Barclay, S. \& Booth, S. (2007). What are patients' priorities for palliative care research? Palliative Medicine, 21, 219-225.

Perkins, P., Booth, S., Vowler, S.L. \& Barclay, S. (2008). What are patients' priorities for palliative care research? A questionnaire study. Palliative Medicine, 22, 7-12.

Ross, C. \& Cornbleet, M. (2003). Attitudes of patients and staff to research in a specialist palliative care unit. Palliative Medicine, 17, 491-497.

Rudolph, S., Hiscock, H., Price, A., Efron, D., Sewell, J., South, M. \& Wake, M. (2009). What research questions matter to Australian paediatricians? National Delphi Study. Journal of Paediatrics and Child Health, 45, 704-710.

Sigurdardottir, K.R., Haugen, D.F., van der Rijt, C.C.D., Sjøgren, P., Harding, R., Higginson, I.J. \& Kaasa, S. on behalf of project PRISMA. (2010). Clinical priorities, barriers and solutions in end-of-life cancer care research across Europe: Report from a workshop. European Journal of Cancer, 46, 1815-1822.

Steele, R., Bosma, H., Johnston, M.F., Cadell, S., Davies, B., Siden, U.H. \& Straatman, L. (2008). Research priorities in pediatric palliative care: A Delphi study. Journal of Palliative Care, 24, 229-287.

Terry, W., Olson, L.G., Ravenscroft, P., Wilss, L. \& Boulton-Lewis, G. (2006). Hospice patients' views on research in palliative care. Internal Medicine Journal, $36,406-413$.

Tieman, J.J., Sladek, R.M. \& Currow, D.C. (2009). Department multiple sources: Mapping the literature of palliative care. Palliative Medicine, 23, 425-431.

Tolley, D.C. \& Payne, R. (2008). Hospice partnerships with academic entities: Philosophical and historical background and assessment of future needs. Palliative Medicine, 22, 921-928.

White, C., Gilshenan, K. \& Hardy, J. (2008). A survey of the views of palliative care healthcare professionals towards referring cancer patients to participate in randomized controlled trials in palliative care. Supportive Care in Cancer, 16, 1397-1405.

White, C. \& Hardy, J. (2010). What do palliative care patients and their relatives think about research in palliative care? A systematic review. Supportive Care in Cancer, 18, 905-911.

Whiting, L.S. \& Vicker, P.S. (2010). Conducting qualitative research with palliative care patients: Applying Hammick's research ethics wheel. International Journal of Palliative Nursing, 16, 58-68.

Wilson, S., Ramelet, A. \& Zuiderduyn, S. (2010). Research priorities for nursing care of infants, children and adolescents: A West Australian Delphi study. Journal of Clinical Nursing, 19, 1919-1928. 